\title{
Serial postoperative donor-derived cell-free DNA in recipients undergoing pancreas transplantation
}

\author{
Hyun-Ji Lee ${ }^{1}$, Byung Hyun Choi ${ }^{2}$, Kyoung-Hwa Shin ${ }^{3}$, In-Suk Kim, Hyoung-Hoi Kim ${ }^{3}$ \\ 1Department of Laboratory Medicine, Pusan National University Yangsan Hospital, Yangsan, Korea
${ }^{2}$ Department of Surgery, Pusan National University Yangsan Hospital, Yangsan, Korea
${ }^{3}$ Department of Laboratory Medicine, Pusan National University Hospital, Busan, Korea
}

Background: Early detection of rejection after organ transplantation is important as it can prevent deterioration of the transplanted organ. With the development of molecular genetic technology, a digital PCR method that can detect with a small amount of DNA has been developed. In this study, donor derived cell-free DNA (ddcfDNA) was measured in pancreatic transplant patients to investigate the correlation with prognosis after organ transplantation.

Methods: We tested DNA of patients from December 2016 to August 2018, comprising seven donors and seven recipients of pancreas transplantation. Single nucleotide polymorphism (SNP) markers were selected to differentiate donor DNA from recipient DNA. Plasma cell-free DNA (cfDNA) is extracted from whole blood and subjected to the parallel quantification of recipient and ddcfDNA, by the use of digital PCR for SNPs different between those two genomes. The donor DNA percentage was calculated as the corrected donor DNA counts divided by the total positive DNA counts, multiplied by 100. Repeated ddcfDNA determinations were scheduled to be performed in the study on postoperative day (POD) 1, 4, 7, 10, and 14 days after transplantation.

Results: All seven patients were insulin-dependent diabetes mellitus, two males and five females. The age of the recipient was median 34 years (range, 26-58 years). The preoperative body mass index was median $23.15 \mathrm{~kg} / \mathrm{m}^{2}$ (range, $15.94-25.61 \mathrm{~kg} / \mathrm{m}^{2}$ ). The preoperative HbA1c was median 8.2 (range, 5.5-12.10). The ddcfDNA (\%) measured serially is median 0.54 (range, $0.24-$ 66.53) for POD 1, median 3.8 (range, 1.72-50) for POD 4, median 1.69 (range, 0-52.94) for POD 7, median 1.22 (range, 0.16-53.84) for POD 10, and median 2.75 (range, 0.38-71.42) for POD 14. During the same period, the values of amylase are median 55 (range, 22-71) for POD1, median 52 (range, 27-77) for POD 4, median 85 (range, 61-139) for POD 7, median 82 (range, 60-147) for POD 10, and median 80 (range, 66-145) for POD 14.

Conclusions: In this study, ddcfDNA was measured for 2 weeks after transplantation in pancreatic transplant recipients.

Corresponding author: Hyun-Ji Lee

E-mail: hilhj1120@gmail.com

(C) The Korean Society for Transplantation

This is an Open Access article distributed under the terms of the Creative Commons Attribution Non-Commercial License (http://creativecommons.org/licenses/by-nc/4.0/) which permits unrestricted non-commercial use, distribution, and reproduction in any medium, provided the original work is properly cited. 